\title{
Cognition and Religious Phenomena - A Response to Håkan Rydving
}

JESPER SØRENSEN

University of Southern Denmark

In the following I address some of the critique of the cognitive science of religion (CSR) raised by Håkan Rydving (HR) in this issue of Temenos. My answer is made from the personal perspective of a scholar engaged in cognitive research into religious phenomena, and should not be regarded as representative of other scholars working within the same tradition.

First I want to thank HR for taking CSR seriously and discussing it from a theoretical and methodological perspective, and to the editors of Temenos for asking me to offer a response to HR's criticism. I find such interest both productive and necessary, in order that the results and perspectives of CSR can be of benefit to the wider scientific study of religion and vice versa. Thus it should be stated from the outset that CSR is not the only relevant perspective that can be taken on religion, and that CSR itself comprises a number of different approaches. The extent to which some of its proponents have been over-enthusiastic in appraisal and dismissive of alternative approaches, as argued by HR, has been discussed within the CSR as well (e.g., A. Geertz 2004), and works combining cognitive theory with more traditional approaches in the social sciences have recently been published (Whitehouse \& Laidlaw 2007). If the style and rhetoric of the proponents of CSR have led some scholars to ignore or outright reject cognitive approaches, this is deplorable; but I strongly suspect that these are not the only reasons, and hopefully HR will at some point direct his hermeneutic of suspicion towards the underlying motives for rejecting the cognitive approach.

Leaving aside matters of sociology of science and expository style, I wish to address some of the more fundamental issues raised by HR and point to what I find to be a number of misunderstandings and misrepresentations. I will address three issues raised by HR: (1) the relationship between interpretation and explanation ('what are we doing'); (2) the concept of 'religion' 
and the subject-matter under investigation ('what are we investigating'); and (3) the (lack of) methodological rigor in CSR in testing hypotheses ('how are we doing $i^{\prime}$ ).

\section{What Are We Doing?}

HR addresses the often-debated relation between explanation and interpretation within the study of human behavior by questioning CSR's underlying reasons for claiming that religion is 'natural'. As well as a wish to explain religion by the methods of natural science, HR more than implies that the main reason for claiming the naturalness of religion is to bolster the prestige of the natural sciences. However, 'natural' as opposed to 'cultural' is first and foremost a rejection of the widespread assumption that religion is a purely 'cultural' phenomenon, and that human biology and evolved cognitive capacities have little or no impact on the emergence and transmission of religious ideas and practices. The latter position has been argued in numerous almost canonical texts within the study of religion, most notably in Clifford Geertz Religion as a Cultural System (1993). As such, the 'cultural / social origin of religion' appears to be the standard paradigm within religious studies, and one which has not been seriously questioned by postmodern or poststructuralist approaches, even if the concepts of religion and culture have been contested. CSR, in contrast, argues that without addressing the role played by human cognitive processing we will not be able to explain the huge number of recurrent phenomena found cross-culturally and throughout history. The focus of CSR is on the cross-culturally recurrent phenomena usually described in terms of the concept of 'religion': ritual behavior, representations of a 'soul', representations of an after-life, representations of superhuman agents, the emergence of religious coalitions and institutions etc. CSR thus resuscitates the almost forgotten 'grand questions' of why humans have 'religion' at all, why religious forms are recurrent in all human societies, and why some particular features and not others seem to reappear in different cultural contexts. It is argued that human cognitive architecture constrains both the emergence and the transmission of religious phenomena and that particular cognitive abilities underlie the creative expansion of religious ideas and practices. CSR's interest in universally recurrent phenomena, and the persuasion that human cognitive mechanisms are at least partly the cause thereof, naturally leads to a more explanatory approach - at least if hypotheses are to be tested and not merely posited. For detailed discussions concerning the relation between interpretation and explanation I refer interested readers to Lawson and McCauley (1990) 
and Sperber (1996). All argue that interpretation is a necessary part of investigating human behavior, both in terms of data gathering and in terms of interpreting results derived from experiments. Interpretation is, as such, an integral part of human cognitive processing. Focusing on more explanatory approaches, however, is both an attempt to right a gross imbalance in favor of interpretations and a firm rejection of a widespread dogma: that phenomena relevant for social scientists can only be understood by means of a hermeneutic process relating observed behavior and human products to its immediate context, whether social or semantic. In contrast it is argued that a substantial amount of human behavior is in fact explicable in terms of universal features of the human cognitive and neurological system. The role of 'culture', i.e. of learning, is more constrained than is believed by the majority of scholars working in the human and social sciences, while the influence of a particular culture on human cognition is a datum that needs to be explained by reference to precise mechanisms rather than posited as an explanation itself. Humans are not born as blank slates; and even though proponents of the CSR disagree about the importance of cultural learning, all more or less agree that the differential transmission of cultural material is something that needs to be explained and that an understanding of evolved cognitive dispositions is central in arriving at adequate answers.

This in itself might have a hegemonic tinge to it, if we do not bear in mind that explanations are always incomplete and call for further investigations (Lawson \& McCauley 1990, 10). Whereas the relevance of an explanatory theory is of course constrained by the extent to which it subsumes empirical observations under a general model, its fruitfulness lies as much in its ability to generate new questions and to further empirical investigations and creative model-building. Furthermore, cognitive and evolutionary theories of religion are strengthened by being embedded in coherent frameworks of theories ranging from evolutionary biology and brain science, over experimental and social psychology, to philosophy of mind, linguistics and economic theory. Theories are thus not only to be judged relative to their own subject material but also to the degree they fit with other scientific theories. Evolutionary biology, neuroscience and cognitive science provide such strong foundations; and while some students of religion apparently see this as a threat to their traditional turf, others see it as a golden opportunity to reinsert the study of religion into the larger framework of the sciences more generally.

Developing explanatory theories of phenomena covered by the concept of 'religion', however, does not merely explain universal features - it also 
provides a crucial input to interpretative endeavors. Interpretations not grounded in or backed by explanatory theories of human 'nature', i.e. species-specific cognitive processing and behavior, are prone either to operate on the basis of implicit assumptions derived from naïve folk psychology or simply to run wild, i.e. to be based on nothing but the researcher's whims. Thus such notions as human agency, memory, causality, modes of categorizing etc. are implicitly or explicitly at work in all analyses of human behavior, whether historical or contemporary. Understanding the role of human cognitive processes is therefore critical both to explaining universally recurrent features of 'religion' and to interpreting local and particular situations and describing historical trajectories. I take it that HR agrees with the gist of this, when he states that he does not oppose 'the obvious fact that cognition is essential to everything we think and do as human beings'. I am afraid, however, that he grossly underestimates the opposition to research programs based on explanatory theories of universal, species-specific traits in human behavior, at least insofar as these 'trespass' into subject areas dominated by hermeneutic approaches. Some representatives of the CSR might be accused of demonstrating a hegemonic style of writing, but the actual positions of power within the scientific study of religion are firmly controlled by non-cognitivists.

Whether CSR has so far succeeded in explaining aspects of religion, or on the contrary has failed in actually fulfilling the promised results, is a question I approach below. The central questions at this point are whether (some) religious phenomena can or should be explained by means of general principles, and whether the scientific investigation of religious phenomena should be integrated with other scientific theories, such as those found in evolutionary biology, psychology and neurology. CSR says yes; traditional humanistic approaches say more or less emphatically no; and HR says...?

\section{What Are We Investigating?}

Explaining religious phenomena of course entails that we have some clue as to what phenomena we are actually investigating. At this point HR comes up with his most serious, but unfortunately also most misguided, critique of CSR. He argues that CSR attributes a folk category, 'religion', to the human mind and thus naturalizes what is essentially a Western concept, a movement that 'amounts to a new form of western imperialism'.

If this were true, it would be a serious problem; but either HR is deliberately misrepresenting arguments from proponents of CSR in order to make a 
polemical point, or his hermeneutic of suspicion has turned into full-fledged paranoia producing non-existing straw men. HR's discussion of the relation between CSR and the concept of religion is unsatisfactory for three reasons. First, proponents of CSR have generally been in line with the postmodern critique of the concept of 'religion' (although for very different reasons). For instance Boyer argues persistently that religion is an 'impure object' that cannot be explained as a whole because it is an improper domain for scientific reasoning (if not for other things). Thus making a theory of 'religion' is no more tractable than making a theory of 'white things' or 'trees'. Even though we can find things in the world that fit with the categories of both 'white things' and 'trees', none of the domains are any good for scientific theorizing. Folk concepts must be fragmented into their constituent parts, thereby relating them to domains more tractable for theorizing (Boyer 1994, 1996, 2001; see also Sørensen 2005). Thus no magical bullet will explain 'religion' because 'religion' is a synthetic concept that pragmatically delineates a range of human behaviors. However, getting rid of 'religion' does not explain (away) the aforementioned behavior - contrary to HR's argument that getting rid of 'religion' would leave CSR 'nothing to explain and the whole enterprise would be futile'.

If religion is a 'bad' concept, why do the proponents of CSR use the concepts of religion at all? This brings me to my second point, which is fundamentally an epistemological discussion of what concepts are and what role they play in the attainment of knowledge. HR has a blind spot, also found among other proponents of the 'hermeneutics of suspicion': they endorse an implicit positivism, according to which scientific concepts should refer to something 'real' in the sense of corresponding to something in the real world. Genealogical studies, however, inevitably 'expose' the fact that the concepts used all have a history outside science, and following this it is argued that this cultural history systematically skews our perception of reality. Therefore, if a concept is found to be too closely related to local conceptual structures, it is suspicious and should be exorcised or at least restricted to use in its culture of origin. 'Religion' obviously has a long history in the 'West' and in relation to Christianity; and as people and languages around the world 'lack' a term referring to this human behavior, we can conclude that it does not refer to the 'real' world but only to a particular cultures notion of such. Contrary to this view, apparently endorsed by HR, psychological investigations into human categorization tell us that categories refer to models of the world, and scientific models are distinct from folk models by their degree of explicitness, the ability of the models to subsume a number of phenomena 
in the world, and their degree of coherence with other models. Thus nothing prevents a concept from referring to both a folk and a scientific model, and transformation from the former into the latter occurs constantly. What constitutes a scientific concept is the extent to which it explicitly refers to an underlying model, in this case a theory of religion, and how this theory fits with other scientific theories. Finally, the fact that some languages do not have a concept of 'religion' can never be an argument against the usefulness of the concept from a scientific point of view. Whether people have a notion of gravity or not does not affect the question whether that concept might provide a useful model of real-world phenomena, and thus be helpful in explaining the physical features of for instance tools belonging to a culture without a local concept corresponding to 'gravity'.

HR's understanding of Lévi-Strauss' critique of the concept of totemism is illustrative of our disagreement. HR argues that Lévi-Strauss' dissolution of 'totemism' as an object of study should be exemplary of a similar dissolution of the concept of religion. What Lévi-Strauss argued, however, was not merely the abandonment of the concept of 'totemism' (in itself a futile quest) but rather that no theory could be made for this 'object'. Instead, in order to be explained the phenomenon referred to had to be reduced to aspects of an underlying mechanism, namely that of human categorization. Lévi-Strauss thus gave an explanation of 'totemism' by reducing it to underlying mechanisms active in other phenomena as well, thereby 're-describing' the phenomenon as something general and natural rather than exotic and mysterious. We see a similarly reductive move in CSR, when for instance anthropomorphic and animistic thinking is explained by reference to universal cognitive mechanisms, such as Theory of Mind or a Hyperactive Agency Detection Device. Universal aspects of religious phenomena have to be reduced to underlying mechanisms in order to be explained; and this effectively means that the explanatory principles will cover more phenomena than those referred to by the concept of 'religion'. Thus, just as Lévi-Strauss and others kept using the concept of totemism as a referential device pointing to a particular range of phenomena, similarly proponents of CSR (as well as most other people) continue to use the concept of 'religion', even though most of us believe that the concepts covers many different phenomena, each in need of explanation. ${ }^{1}$

1 In this context it is ironic that Lévi-Strauss argues something similar to CSR concerning the concept of 'religion': 'Conversely, if religious ideas are accorded the same value as any other conceptual system, as giving access to mechanisms of thought, the procedure of religious anthropology will acquire validity, but it will lose its autonomy and specific character'. (Lévi-Strauss 1991, 104.) 
Thirdly, HR wants to have his cake and eat it. At the same time that he (wrongly) accuses CSR of reifying and naturalizing the concept of religion as a property of the brain, he criticizes particular experiments (Barrett \& Nyhof 2001; Boyer \& Ramble 2001) for not really testing 'religious concepts'. Thus not only does HR introduce double standards (he can use the concept of 'religion' to denote a particular range of concept, but CSR cannot) - he also misses the whole point of the study. Boyer and Ramble do the opposite of reifying 'religion': they operationalize the concept in order to test particular hypotheses! Based on a number of studies in experimental, developmental and cross-cultural psychology, Boyer claims that humans have a number of intuitive expectations for things as these are processed by different cognitive systems. 'Intuitive' is a technical term that refers to universal features of human categorization that are independent of cultural input (contrary to HR's misrepresentation of the theory). Based upon this, Boyer has proposed the hypothesis that the concepts we call 'religious' (amongst other things) consist of a particular combination of intuitive and counterintuitive properties, and that this mix is cognitively optimal in that it enhances both memorability and transmission. The concepts we refer to as 'religious' thus have an advantage in transmission and will be prevalent in all cultural settings. One might question the underlying theory of human categorization, as well as Boyer's general theory of religious concepts. However, the experiment criticized does not test these theories directly, but rather 'the predictions of a particular cognitive account of religious concepts' (Boyer \& Ramble 2001, 536; emphasis added), i.e. whether concepts with a particular combination of intuitive and counterintuitive features are better recalled than other concepts. In fact, Boyer and Ramble had to construct new artificial concepts in order to prevent differences in recall from being explicable by prior exposure.

In sum: HR seems strangely ambivalent about what to do with the concept of 'religion'. On the one hand he wants to get rid of a concept that he believes forms part of western conceptual imperialism; on the other he seems to argue that there is a special category of concepts in any culture that is 'religious'. He effectively ends up essentializing the concept from a culturalist perspective: every culture has its own 'religious' dimension, but this cannot be circumscribed by means of the local concept of religion - thus the rhetorical call for a cognitive science of dharma or dîn. Furthermore, HR is very concerned that CSR be relegated to its proper place in the study of religion along with other approaches. What remains a mystery, however, is why HR is so concerned to protect multiple approaches to a field that has no object. 


\section{How Are We Doing It?}

This brings me to my final point, concerning the experimental dimension of CSR. I will not enter into a discussion of particular experimental designs, as this would be too lengthy for a reply and is better left to the experimenters themselves. I am personally not satisfied with all experimental designs proposed so far, nor do I always agree with the interpretations of the results. Criticism of particular designs and interpretations should be encouraged, as this is the primary way to improve future research, and as such HR's critique of various experiments is welcome. However, HR extends this to a criticism of more of less all experiments performed within CSR, and in doing so points to two more fundamental problems: that of cross-cultural experimentation and that of the role of falsification.

HR rightly points to the fact that more cross-cultural research is needed in order to test a particular hypothesis proposed by CSR. In fact, several projects are currently under way that are aimed precisely at devising crosscultural tests of various hypotheses both of CSR and of cognitive anthropology more generally. This, however, is more difficult than it sounds. HR suggests that the solution is 'breaking down hypotheses into elements that could be examined by means of carefully formulated questions which would need to be translated into some fifty different languages and implemented in interviews in fifty or more cultural settings'. This suggestion, however, is at best naïve and points to a lack of knowledge of experimental designs and the contextual variables that influence participants' behavior in experimental settings. Few if any of the hypotheses proposed within CSR can be explored by means of simple questionnaires, and translation gives rise to numerous problems.

Further, even though the quality and extent of cross-cultural testing should be improved, HR ignores the fact that many of the hypotheses constructed within CSR are based upon a substantial corpus of experimentally based knowledge obtained in for instance experimental cognitive psychology, developmental psychology, psychopathology and cognitive neuroscience. A number of these studies point to the existence of species-specific cognitive mechanisms not explicable by cultural input. One can of course criticize the extent to which these tests have been sufficiently controlled for cultural variance, but once again suspicion should not turn into paranoia: no matter how many tests are conducted worldwide, it can always be claimed that this is not sufficiently cross-cultural. There are good reasons, however, to believe that a substantial number of cognitive abilities are species-specific rather than culture-specific. In so far as theories within CSR test hypotheses 
based on findings of such universal cognitive mechanisms, there is no a priori reason to test them cross-culturally. HR's argument is therefore directed as much against the cognitive science community in general as against CSR alone. This is a debate which has been going on for a long time and is likely to continue. This is obviously by no means to say that cross-cultural testing is not important. Such experiments, however, need to test particular hypotheses, predicting specific effects of cultural learning. It is not enough simply to state that 'culture matters'. This has to be pinned down in the form of claims and hypotheses that can be operationalized in concrete experimental designs. HR mentions the role of education as a variable not taken into account, but he fails to present any reasons why this would change the results of the experiments discussed. He merely asserts that this is the case. The great thing about science, however, is that experiments can eventually be conducted that test precisely such variables. My own experience is that proponents of CSR would be delighted to produce results that went against common theories and contrary to HR's claim, the aspects of Boyer and Rambles (2001) results that indicate cultural effects on memorability of counterintuitive concepts have been widely discussed. Further, a number of studies by Tweney, Slone, Upal and colleagues have been specifically aimed at testing central components of Boyer's counterintuitive hypothesis (Slone et al. 2007; Upal et al. 2007). The problem we face is that few of us within the study of religion have been trained in experimental methods; some of our experiments might therefore fail to live up to standards. Hopefully this will change in the near future, and empirical studies of the role of cultural learning are likely to gain ground, as the role of culture is one of the major objects of contention within CSR itself.

This brings me to my second point, namely HR's notion of falsification. Again the 'hermeneutics of suspicion' reveals an implicit positivism by defending a naïve 'falsificationism' as the basic epistemology of the natural sciences. HR's position was even stronger when he gave the paper as a plenary lecture at the IAHR's regional conference in Stockholm in 2007. At that time chemistry was presented as the model science whose experimental standards CSR should live up to in order for its claims to have any kind of validity. One cannot help wondering whether such disciplines as for instance experimental psychology or medicine would live up to the standards of chemistry. Working with human participants necessarily imposes certain constraints on experimental designs not found when working with lifeless molecules. Even in the case of the 'hard' natural sciences I suspect that HR's epistemology represents an outdated positivism. No one sets up experiments simply to 
falsify their own theories. This is in general better left to others within the scientific community - scientists who will often have theoretical interests in disproving their colleagues' theories. Of course experiments should be created so as to possibly falsify a hypothesis; but experiments specifically test hypotheses built upon theories and not the theories themselves. Thus even if a hypothesis is falsified it does not automatically follow that the theory is invalid. The hypothesis derived from the theory might be wrong, or the means of operationalizing the hypothesis in the experiment might be flawed. Falsifying a hypothesis entails that the underlying theory is weakened, as one of its attempts to produce a falsifiable but true hypothesis about the world has failed, and due to the fact that one might need to build ad hoc hypotheses to explain the experimental results. The theory itself, however, should only be given up if a competing theory presents a better explanation. So far it is hard to find competing theories within the study of religion more amenable to falsification than those endorsed by proponents of CSR.

In sum: the problem with the 'strong falsificationism' apparently endorsed by HR (at least for the natural sciences) is that it is a normative description, remote from the concrete behavior of scientists, and that it ignores the fact that attempts to falsify usually stem from competing underlying theories.

Why would HR use such a radical version of natural science epistemology to evaluate CSR? Why not make experimental psychology, or cognitive science, the model - considering that most findings in CSR, after all, are published in peer-reviewed journals within these areas? One reason may be that HR has a general suspicion as to whether these fields produce any valid claims, i.e. he does not consider them to be 'proper sciences' like mathematics, physics and chemistry. A more sinister possibility would be that comparing CSR to a 'hard' science such as chemistry effectively insulates the traditional study of religion from criticism based on the cognitive approach by offering the off-hand rejection that 'CSR does not live up to its own standards' and therefore need not be taken seriously. I am thus not at all certain that HR's critical review will have the desired effect of fertilizing discussions between proponents of CSR and the rest of the academia of religion. 


\section{Bibliography}

\section{Barrett, Justin L. \& Melanie A. Nyhof}

2001 Spreading Non-natural Concepts: The Role of Intuitive Conceptual Structures in Memory and Transmission of Cultural Materials. - Journal of Cognition E Culture 1, 69-100.

\section{Boyer, Pascal}

1994 The Naturalness of Religious Ideas: A Cognitive Theory of Religion. Berkeley: University of California Press.

1996 Religion as an impure object-Method and Theory in the Study of Religion 8(2), 201-13

2001 Religion Explained: The Human Instincts that Fashions Gods, Spirits and Ancestors. London: Vintage.

\section{Boyer, Pascal \& Charles Ramble}

2001 Cognitive templates for religious concepts: cross-cultural evidence for recall of counter-intuitive representations - Cognitive Science 25, 535-64.

Geertz, Armin W.

2004 Cognitive approaches to the study of religion. - Peter Antes \& Armin W. Geertz \& Randi R. Warne (eds), New Approaches to the Study of Religion. 2. Textual, Comparative, Sociological, and Cognitive Approaches (Religion and Reason 43), 347-99. Berlin: Walter de Gruyter.

\section{Geertz, Clifford}

1993 (1973) Religion as a Cultural System - Clifford Geertz, The Interpretation of Cultures, 87-125. London: Fontana Press.

\section{Lawson, E. Thomas \& Robert N. McCauley}

1990 Rethinking Religion: Connecting Cognition and Culture. Cambridge: Cambridge University Press.

\section{Lévi-Strauss, Claude}

1991 (1962) Totemism. Translated by Rodney Needham. London: Merlin Press.

\section{Slone, D. Jason \& Lauren Gonce \& M. Afzal Upal \& Kristin Edwards \&} Ryan Tweney

2007 Imagery effects on recall of minimally counterintuitive concepts - Cognition and Culture 7(3-4), 355-67.

\section{Sperber, Dan}

1996 Explaining Culture: A Naturalist Approach. Oxford: Blackwell Publishers. 


\section{Sørensen, Jesper}

2005 Religion in Mind: A Review Article of the Cognitive Science of Religion - Numen: International Review for the History of Religions 52(4), $465-94$.

Upal, M. Azfal \& Lauren Gonce \& Ryan Tweney \& D. Jason Slone

2007 Contextualizing counterintuitiveness: How context affects comprehension and memorability of counterintuitive concepts - Cognitive Science 31(3), 415-39.

Whitehouse, Harvey \& James Laidlaw

2007 Religion, Anthropology and Cognitive Science. Durham: University of Carolina Press. 